\title{
THE VOLUME OF THE FUNDAMENTAL DOMAIN FOR SOME INFINITE GROUPS*
}

\author{
BY \\ CARL LUDWIG SIEGEL
}

Let $Q$ be any region in $m$-dimensional euclidean space which is invariant under a group $\Gamma$ of real homogeneous linear transformations of the coordinates. The group $\Gamma$ has a fundamental domain $F$ on $Q$ if $F$ is mapped by the different transformations of $\Gamma$ into a set of domains which completely fill out $Q$ without overlapping one another. It is obvious that then $\Gamma$ is countable. If all the substitutions of the group have the determinant \pm 1 , the volume $v$ of $F$ is uniquely determined by $Q$ and $\Gamma$. The reciprocal value of $v$ is a certain measure for the order of $\Gamma$; in fact, if $\Gamma_{1}$ is a subgroup of $\Gamma$ with the index $g$, the volume of the fundamental domain of $\Gamma_{1}$ is exactly $g v$.

It is known from the analytic theory of quadratic forms how to find $v$ if $\Gamma$ is the group of automorphisms of a quadratic form with integer coefficients. Minkowski, in his last investigations on the theory of numbers, determined the value of $v$ in another case, which also has interesting applications to the problem of the closest packing of $n$-dimensional spheres. Let $\sum_{k, l=1}^{n} s_{k l} x_{k} x_{l}$ be any positive definite quadratic form of $n$ variables and $Q$ that part of the space of the $n(n+1) / 2$ coefficients $s_{k l}(1 \leqq k \leqq l \leqq n)$ where the determinant $\left|s_{k l}\right|$ is not greater than a fixed positive number $q$. By applying any substitution $x_{k}=\sum_{l=1}^{n} c_{k l} y_{l}$ with integer coefficients whose determinant is \pm 1 , a linear transformation of the $s_{k l}$ is induced which leaves $Q$ invariant. The group $\Gamma$ of these transformations of the quadratic form is obviously isomorphic to the factor-group of the group of all unimodular substitutions of $n$ variables with respect to the subgroup of order 2 generated by $x_{k}=-y_{k}(k=1, \cdots, n)$. A fundamental domain of $\Gamma$ on $Q$ is the region $F$ of the reduced positive definite quadratic forms of $n$ variables whose determinant is not greater than $q$. Minkowski proved that $F$ is bounded by a finite number of planes and the surface $\left|s_{k l}\right|=q$. Moreover, he calculated explicitly the volume of $F$ as a function of $n$ and $q$, namely,

$$
v=\frac{2}{n+1} q^{(n+1) / 2} \pi^{-n(n+1) / 4} \Gamma\left(\frac{1}{2}\right) \Gamma\left(\frac{2}{2}\right) \cdots \Gamma\left(\frac{n}{2}\right) \zeta(2) \cdots \zeta(n),
$$

where $\zeta(s)$ denotes the zeta function of Riemann.

\footnotetext{
* Presented to the Society, October 26, 1935; received by the editors April 1, 1935.
} 
The purpose of the present paper is to prove Minkowski's formula (1) by a simple analytic method and to generalize it to the case of any algebraic number field. A special application gives the non-euclidean volume of the fundamental domain for the modular group in every totally real algebraic field. Blumenthal and Hecke have shown the importance of the corresponding modular functions for algebraic and arithmetic investigations. Since the knowledge of a set of generators of the modular group is necessary for the construction of any example in the theory of modular functions, the determination of the volume of the fundamental domain can be useful for further researches.

1. Let $Q_{0}$ be the space of all positive definite symmetric matrices $\mathfrak{X}$ of $n$ rows, and $F_{0}$ its fundamental domain for the group of all transformations $\mathfrak{C}^{\prime} \mathfrak{X} \mathfrak{E}$ where $\mathfrak{E}$ is any unimodular matrix of $n$ rows and $\mathfrak{C}^{\prime}$ its transposed. The trace of $\mathfrak{X}$ is denoted by $\sigma(\mathfrak{X})$, the determinant of $\mathfrak{X}$ by $|\mathfrak{X}|$, and $d \mathfrak{X}$ is the $\frac{1}{2} n(n+1)$-dimensional volume element in $Q_{0}$. The formula

$$
\prod_{k=0}^{n-1} \pi^{-(s+k) / 2} \Gamma\left(\frac{s+k}{2}\right)=\int_{Q_{0}}|\mathfrak{X}|^{s / 2-1} e^{-\pi \sigma(\mathfrak{X})} d \mathfrak{X}
$$

holds for every $s$ with positive real part and can be proved by complete induction, starting with Euler's definition of the gamma function. Let $\mathfrak{A}$ be any real matrix of $n$ rows and columns, whose determinant is not zero. Then $\mathfrak{A}^{\prime} \mathfrak{X} \mathfrak{A}$ can be substituted for $\mathfrak{X}$ in (2). Hence

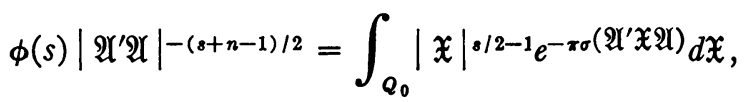

where $\phi(s)$ is an abbreviation for the left side of (2).

If $\mathfrak{X}$ runs over the fundamental domain $F_{0}$ and $\mathfrak{E}$ over all unimodular matrices of $n$ rows, the matrices $\mathfrak{E}^{\prime} \mathfrak{X} \mathfrak{C}=(-\mathfrak{E})^{\prime} \mathfrak{X}(-\mathfrak{C})$ completely fill out twice the space $Q_{0}$. Therefore (3) can be transformed into the equation

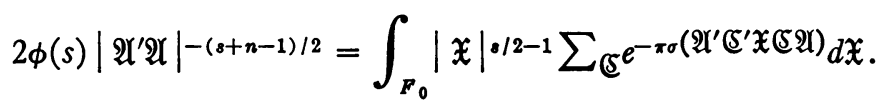

A matrix $\mathfrak{B}$ is called associated to $\mathfrak{A}$, if $\mathscr{B}=\mathfrak{C} \mathfrak{A}$ with unimodular $\mathfrak{E}$. In (4), the matrix $\mathfrak{E} \mathfrak{A}$ runs over all associates to $\mathfrak{A}$. It is clear that the determinants of all associates have the same absolute value. There exist only a finite number of non-associate integer matrices $\mathfrak{A}$ whose determinants have a fixed absolute value $a \neq 0$. In fact, Eisenstein has proved that their number is

$$
\psi(a)=\sum a_{1}{ }^{n-1} a_{2}{ }^{n-2} \cdots a_{n-1}{ }^{1} a_{n}{ }^{0},
$$


where $a_{1}, \cdots, a_{n}$ run over all systems of solutions of $a_{1} \cdots a_{n}=a$ in positive integers.

Let the real part of $s$ be greater than 1 and sum (4) over a complete system of non-associated integer matrices $\mathfrak{A}$ whose determinants are different from zero. Since

$$
\sum_{a=1}^{\infty} \psi(a) a^{-s-n+1}=\zeta(s) \zeta(s+1) \cdots \zeta(s+n-1),
$$

the result is

(6) $2 \phi(s) \zeta(s) \zeta(s+1) \cdots \zeta(s+n-1)=\int_{F_{0}}|\mathfrak{X}|^{\cdot / 2-1} \sum_{|\mathfrak{X}| \neq 0} e^{-\pi \sigma\left(\mathfrak{I}^{\prime} \mathfrak{x} \mathfrak{X}\right)} d \mathfrak{X}$,

where $\mathfrak{A}$ runs over all integer matrices with $|\mathfrak{A}| \neq 0$.

The left side of (6) is a meromorphic function of $s$ which has a pole of first order at $s=1$. The residue at this pole is

$$
\rho=2 \pi^{-n(n+1) / 4} \Gamma\left(\frac{1}{2}\right) \Gamma\left(\frac{2}{2}\right) \cdots \Gamma\left(\frac{n}{2}\right) \zeta(2) \cdots \zeta(n) .
$$

To study the behavior of the right side of (6) near $s=1$, the well known method from the theory of the zeta functions can be used. Divide $F_{0}$ into two parts $F_{1}$ and $F_{2}$, corresponding to $|\mathfrak{X}| \leqq 1$ and $|\mathfrak{X}|>1$. The integral in (6) then splits up into the sum of the two integrals over $F_{1}$ and $F_{2}$. The second integral is an integral function of $s$. Furthermore the function

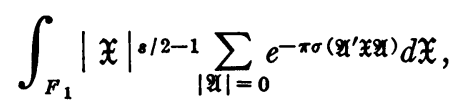

where $\mathfrak{A}$ runs over all integer matrices with $|\mathfrak{A}|=0$, is regular near $s=1$. Hence $\rho$ is also the residue of

$$
\int_{F_{1}}|\mathfrak{X}|^{\mid s / 2-1} \sum_{\mathfrak{X}} e^{-\pi \sigma\left(\mathfrak{X}^{\prime} \mathfrak{x}\right)} d \mathfrak{X}
$$

where $\mathfrak{A}$ runs over all integer matrices, at the point $s=1$. Now, from the theory of theta functions, the formula

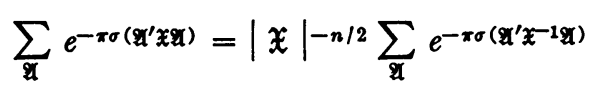

is known. Hence $\rho$ is the residue of

$$
\int_{F_{1}}|\mathfrak{X}|^{(s-n) / 2-1} d \mathfrak{X}+\int_{F_{1}}|\mathfrak{X}|^{(s-n) / 2-1} \sum_{\mathfrak{X}}^{\prime} e^{-\pi \sigma\left(\mathfrak{X}^{\prime} \mathfrak{X}^{-1 \mathfrak{X})}\right.} d \mathfrak{X},
$$


where $\mathfrak{A}$ runs over all integer matrices except the zero matrix. The second integral in (8) is again regular at $s=1$, and $\rho$ is the residue of the first integral in (8).

If $v_{1}$ is the volume of $F_{1}$, the fundamental domain $F$, which is the part $|\mathfrak{X}| \leqq q$ of $F_{0}$, has the volume $v_{1} q^{(n+1) / 2}$. Hence

$$
\begin{gathered}
\int_{F_{1}}|\mathfrak{X}|^{(s-n) / 2-1} d \mathfrak{X}=\frac{n+1}{2} v_{1} \int_{0}^{1} q^{(s-n) / 2-1} \cdot q^{(n-1) / 2} d q=\frac{n+1}{s-1} v_{1}, \\
\rho=(n+1) v_{1},
\end{gathered}
$$

and (1) follows from (7) and (9).

2. The group of the matrices (5) with integer rational elements and the determinant \pm 1 has a generalization in any algebraic number field. It consists of all matrices $\mathbb{S}$ of $n$ rows, for which the elements of $\mathbb{S}$ and $\mathfrak{S}^{-1}$ are integers of the field $K$. These matrices will be called unimodular in $K$. Their determinants are units of the field $K$. The definition of the associates of a matrix can be at once extended to the case of any $K$ : the matrix $\mathfrak{B}$ is associated to $\mathfrak{A}$, if $\mathfrak{B}=\mathfrak{E} \mathfrak{A}$ with a unimodular $\mathfrak{E}$ in $K$. Since the determinant of an associate of $\mathfrak{A}$ can only differ from the determinant $|\mathfrak{A}|=\alpha$ by a factor which is a unit of $K$, the determinants of all associates to an integer matrix of $K$ define the same principal ideal ( $\alpha$ ). Eisenstein's result (5) has been generalized by Hurwitz. He proved that the number of non-associate integer matrices $\mathscr{A}$ of $K$ with $n$ rows, whose determinants $\alpha \neq 0$ define the same principal ideal $(\alpha)$, is

$$
\psi(\alpha)=\sum N\left(\mathfrak{a}_{1}{ }^{n-1} \mathfrak{a}_{2}{ }^{n-2} \cdots \mathfrak{a}_{n-1}^{1} \mathfrak{a}_{n}^{0}\right),
$$

where the symbol $N$ denotes the norm and $\mathfrak{a}_{1}, \cdots, \mathfrak{a}_{n}$ run over all systems of solutions of $\mathfrak{a}_{1} \cdots \mathfrak{a}_{n}=(\alpha)$ in integer ideals $\mathfrak{a}_{1}, \cdots, \mathfrak{a}_{n}$.

In this section only the simpler case of a totally real field $K$ will be investigated. If $l$ is the degree of $K$, the $l$ conjugates of any matrix $\mathfrak{A}$ with elements of $K$ will be denoted by $\mathfrak{A}_{1}, \cdots, \mathfrak{A}_{l}$. Let $\mathfrak{X}_{1}, \cdots, \mathfrak{X}_{l}$ be any $l$ positive definite symmetric matrices of $n$ rows, $Q_{0}$ the space of their $\frac{1}{2} n(n+1) l$ coefficients, and $Q$ the part of $Q_{0}$ defined by the inequality $\left|\mathfrak{X}_{1} \cdots \mathfrak{X}_{l}\right| \leqq q$. If $\mathbb{E}$ is unimodular, the transformation $\mathfrak{E}_{1}^{\prime} \mathfrak{X}_{1} \mathfrak{E}_{1}, \cdots, \mathfrak{E}_{l}^{\prime} \mathfrak{X}_{l} \mathfrak{E}_{l}$ leaves $Q$ invariant. The problem is to prove the existence of a fundamental domain $F$ on $Q$ with respect to these transformations, which is bounded by a finite number of planes and the surface $\left|\mathfrak{X}_{1} \ldots \mathfrak{X}_{l}\right|=q$ and to calculate the volume of $F$. The first part of the problem requires the theory of reduction of positive definite quadratic forms in $K$ and can be solved without serious difficulty by generalizing Minkowski's ideas. Here only the solution of the second part, the determination of the volume $v$ of $F$ by analytic methods, will be explained in detail. 
From (3) there follows for every integer matrix $\mathfrak{A}$ of $K$ with $n$ rows, whose determinant does not vanish, the equation

$$
\phi^{l}(s) N\left(\mathfrak{X} \mathfrak{X}^{\prime} \mathfrak{X}\right)^{-(s+n-1) / 2}=\int_{Q_{0}} N(\mathfrak{X})^{s / 2-1} e^{-\pi S\left(\mathfrak{X}^{\prime} \mathfrak{X} \mathfrak{X}\right)} d \mathfrak{X}_{1} \cdots d \mathfrak{X}_{l} ;
$$

here $N(\mathfrak{X})$ denotes the product of the determinants of $\mathfrak{X}_{1}, \cdots, \mathfrak{X}_{l}$ and $S\left(\mathfrak{A}^{\prime} \mathfrak{X} \mathfrak{X}\right)$ the sum of the traces of $\mathfrak{A}_{1}^{\prime} \mathfrak{X}_{1} \mathfrak{A}_{1}, \cdots, \mathfrak{A}_{l}^{\prime} \mathfrak{X}_{l} \mathfrak{A}_{l}$. If $F_{0}$ is a fundamental domain on $Q_{0}$, the right side of (11) can be transformed in analogy to (4). By summing (11) over a complete system of non-associated integer matrices $\mathfrak{A}$ of $K$ with $|\mathfrak{A}| \neq 0$, the equation

$$
2 \phi^{l}(s) \sum_{(\alpha)} \psi(\alpha) N(\alpha)^{-s-n+1}=\int_{F_{0}} N(\mathfrak{X})^{s / 2-1} \sum_{|\mathscr{X}| \neq 0} e^{-\pi S\left(\mathfrak{I}^{\prime} \mathfrak{X}\right)} d \mathfrak{X}_{1} \cdots d \mathfrak{X}_{\imath}
$$

arises, when $(\alpha)$ runs over all integer principal ideals and $\mathfrak{A}$ over all integer matrices with $|\mathfrak{A}| \neq 0$; the real part of $s$ must be greater than 1 .

If $h$ is the class-number of $K$, there exist exactly $h$ different characters $\chi(\mathfrak{a})$ of the class-group. The sum $\sum_{\chi} \chi(\mathfrak{a})$ is $h$, if $\mathfrak{a}$ is a principal ideal, and 0 otherwise. Let

$$
\zeta_{x}(s)=\sum_{\mathfrak{a}} \chi(\mathfrak{a}) N \mathfrak{a}^{-s}
$$

denote Dedekind's zeta function with class-characters. Then, by (10),

$$
\sum_{(\alpha)} \psi(\alpha) N(\alpha)^{-s-n+1}=h^{-1} \sum_{\chi} \zeta_{\chi}(s) \zeta_{\chi}(s+1) \cdots \zeta_{\chi}(s+n-1) .
$$

Now it is known that $\zeta_{\chi}(s)$ is an integral function if $\chi$ is not the principal character. For the principal character, $\zeta_{x}(s)$ is the function

$$
\zeta_{k}(s)=\sum_{\mathfrak{a}} N \mathfrak{a}^{-s},
$$

which is regular for $s \neq 1$ and has at $s=1$ a pole of first order with the residue $2^{l-1} D^{-1 / 2} R h$, where $R$ and $D$ are regulator and discriminant of $K$. Hence the residue of the left side of (12) at $s=1$ is

$$
\rho=2^{l} D^{-1 / 2} R \prod_{k=1}^{n} \pi^{-k l / 2} \Gamma^{l}\left(\frac{k}{2}\right) \cdot \zeta_{k}(2) \zeta_{k}(3) \cdots \zeta_{\star}(n) .
$$

The calculation of the residue of the right side of (12) is quite analogous to the rational case. The domain $F_{0}$ is divided into the two parts $N(\mathfrak{X}) \leqq 1$ and $N(\mathfrak{X})>1$ and for the first part of $F_{0}$ the theta formula

$$
\sum_{\mathfrak{A}} e^{-\pi S\left(\mathfrak{I}^{\prime} \mathfrak{X} \mathfrak{X}\right)}=D^{-n^{2} / 2} N(\mathfrak{X})^{-n / 2} \sum_{\mathfrak{D}^{-1} \mid \mathfrak{O}} e^{-\pi S\left(\mathfrak{B}^{\prime} \mathfrak{X}-1 \mathfrak{O}\right)}
$$


is used; here $b$ denotes the fundamental ideal of $K$, the matrix $\mathfrak{A}$ runs over all integer matrices of $K$ and $\mathscr{B}$ over all matrices whose elements belong to the ideal $b^{-1}$. In this manner it can be seen that

$$
\rho=(n+1) D^{-n^{2} / 2} v_{1},
$$

where $v_{1} q^{(n+1) / 2}$ is the volume of $F$.

Hence, by (14) and (15), the volume of the fundamental domain $F$ is

$$
v=\frac{2^{l}}{n+1} q^{(n+1) / 2} D^{\left(n^{2} \div 1\right) / 2} R \pi^{-n(n+1) l / 4} \Gamma^{l}\left(\frac{1}{2}\right) \cdots \Gamma^{l}\left(\frac{n}{2}\right) \zeta_{k}(2) \cdots \zeta_{k}(n),
$$

and this is the generalization of Minkowski's formula (1) to the case of any totally real algebraic number field.

3. If some of the conjugates of $K$ are imaginary, let $2 r_{2}$ be their number and $r_{1}$ the number of the real conjugates. Then $r_{1}+2 r_{2}=l$. For any matrix $\mathfrak{A}$ with elements of $K$, the conjugates in the real fields will be denoted by $\mathfrak{A}_{1}, \cdots, \mathfrak{A}_{r_{1}}$ and the conjugates in the imaginary fields by $\mathfrak{A}_{r_{1+1}}, \cdots, \mathfrak{A}_{l}$; moreover $\mathfrak{A}_{k}$ and $\mathfrak{A}_{k+r_{1}}\left(k=r_{1}+1, \cdots, r_{1}+r_{2}\right)$ shall be conjugate complex. Put $r_{1}+r_{2}=p$. Instead of the $l$ positive definite symmetric matrices $\mathfrak{X}_{1}, \cdots, \mathfrak{X}_{l}$ of the totally real case, $r_{1}$ positive definite symmetric matrices $\mathfrak{X}_{1}, \cdots, \mathfrak{X}_{r_{1}}$ and $r_{2}$ positive definite Hermitian matrices $\mathfrak{X}_{r_{1+1}}, \cdots, \mathfrak{X}_{p}$ must be considered. The elements of $\mathfrak{X}_{1}, \cdots, \mathfrak{X}_{p}$ define a space of $n(n+1) r_{1} / 2+n^{2} r_{2}$ real dimensions. Let $Q$ be the part of $Q_{0}$, where $\left|\mathfrak{X}_{1} \cdots \mathfrak{X}_{r_{1}} \mathfrak{X}^{2}{ }_{1+1} \cdots \mathfrak{X}_{p}{ }^{2}\right| \leqq q$. If $\overline{\mathbb{E}}$ denotes the conjugate complex to $\mathfrak{E}$, the transformation $\overline{\mathbb{E}}_{k}^{\prime} \mathfrak{X}_{k} \mathfrak{E}_{k}(k=1, \cdots, p)$ leaves $Q$ invariant for any unimodular matrix $\mathbb{E}$ of the field $K$. The existence of a fundamental domain $F$ on $Q$ for the group of these transformations, which is bounded by a finite number of analytic surfaces, is known for the case of an imaginary quadratic field $K$ by the investigations of Picard and Bianchi. The proof can be extended to the case of any $K$.

For the calculation of the volurne $v$ of $F$, an analogue of (2) for the space $H$ of the positive definite Hermitian matrices $\mathfrak{X}$ must be considered. If $y_{k \lambda}$ and $z_{\boldsymbol{\alpha} \lambda}$ denote real and imaginary parts of the element $x_{\boldsymbol{k} \lambda}$ of $\mathfrak{X}$, the volume element $d \mathfrak{X}$ of $H$ will be defined by

$$
d \mathfrak{X}=\prod_{\kappa, \lambda=1}^{n} d x_{\kappa \lambda}=2^{n(n-1) / 2} \prod_{1 \leqq \kappa \leqq \lambda \leqq n} d y_{\kappa \lambda} \prod_{1 \leqq \kappa<\lambda \leqq n} d z_{\kappa \lambda} .
$$

Then the analogue of (2) is the formula

$$
\prod_{k=0}^{n-1}(2 \pi)^{-s-k} \Gamma(s+k)=\int_{H}|\mathfrak{X}|^{-s-1} e^{-2 \pi \sigma(\mathfrak{X})} d \mathfrak{X} .
$$


If $\mathfrak{X}_{k}(k=p+1, \cdots, l)$ denotes the conjugate complex $\overline{\mathfrak{X}}_{k-r_{2}}$ to $\mathfrak{X}_{k-r_{2}}$, the generalization of (11) is

$$
\begin{gathered}
\left\{\prod_{k=0}^{n-1} \pi^{-(s+k) / 2} \Gamma\left(\frac{s+k}{2}\right)\right\}^{r_{1}}\left\{\prod_{k=0}^{n-1}(2 \pi)^{-s-k} \Gamma(s+k)\right\}^{r_{2}} N\left(\overline{\mathfrak{X}^{\prime}} \mathfrak{X}\right)^{-(s+n-1) / 2} \\
=\int_{Q_{0}} N(\mathfrak{X})^{s / 2} e^{-\pi S\left(\overline{\mathfrak{X}}^{\prime} \mathfrak{X}^{\prime}\right)} \frac{d \mathfrak{X}_{1}}{\left|\mathfrak{X}_{1}\right|} \cdots \frac{d \mathfrak{X}_{p}}{\left|\mathfrak{X}_{p}\right|} .
\end{gathered}
$$

Since the equations $\overline{\mathfrak{E}}_{k}^{\prime} \mathfrak{X}_{k} \mathfrak{\complement}_{k}=\mathfrak{X}_{k}(k=1, \cdots, p)$ only hold identically in $\mathfrak{X}_{k}$ for a unimodular $\mathbb{E}$ of $K$, if $\mathbb{E}=\omega \mathbb{E}$, where $\mathbb{E}$ is the unit-matrix and $\omega$ a root of unity, the matrices $\overline{\mathbb{E}}_{k}^{\prime} \mathfrak{X}_{k} \mathbb{\complement}_{k}(k=1, \cdots, p)$ completely fill out $Q_{0}$ exactly $w$ times, if $\mathfrak{X}_{1}, \cdots, \mathfrak{X}_{p}$ run over the fundamental domain $F_{0}$ and $\mathfrak{E}$ over all unimodular matrices; here $w$ denotes the number of roots of unity in $K$. Hence corresponding to (12) and (13)

$$
\begin{aligned}
& \frac{w}{h} \prod_{k=0}^{n-1}\left\{2^{-r_{2}(s+k)} \pi^{-l(s+k) / 2} \Gamma^{r_{1}}\left(\frac{s+k}{2}\right) \Gamma^{r_{2}}(s+k)\right\} \\
& \cdot \sum_{x} \zeta_{X}(s) \zeta_{X}(s+1) \cdots \zeta_{X}(s+n-1) \\
& \quad=\int_{F_{0}} N(\mathfrak{X})^{s / 2} \sum_{|\mathscr{X}| \neq 0} e^{-\pi S\left(\overline{\mathfrak{A}}^{\prime} \mathfrak{X}\right)} \frac{d \mathfrak{X}_{1}}{\left|\mathfrak{X}_{1}\right|} \cdots \frac{d \mathfrak{X}_{p}}{\left|\mathfrak{X}_{p}\right|},
\end{aligned}
$$

and by calculating the residues at $s=1$ on both sides,

$$
\begin{aligned}
& \frac{2^{r_{1}+r_{2}}}{n+1} q^{(n+1) / 2} D^{\left(n^{2}-1\right) / 2} R \pi^{r_{2}-n(n+1) l / 4} 2^{-n(n+1) r_{2} / 2} \prod_{k=1}^{n} \Gamma^{r_{1}}\left(\frac{k}{2}\right) \Gamma^{r_{2}}(k) \\
& \cdot \zeta_{k}(2) \cdots \zeta_{k}(n)=\int_{F}\left|\mathfrak{X}_{r_{1}+1} \cdots \mathfrak{X}_{p}\right| d \mathfrak{X}_{1} \cdots d \mathfrak{X}_{p},
\end{aligned}
$$

where $D$ is the absolute value of the discriminant of $K$ and $R$ the regulator. Therefore (17) gives the volume of the fundamental domain if the volume element is defined by $\left|\mathfrak{X}_{r_{1}+1} \ldots \mathfrak{X}_{p}\right| d \mathfrak{X}_{1} \ldots d \mathfrak{X}_{p}$ which is invariant under unimodular transformation.

4. The special case $n=2$ is closely connected with the theory of the modular group in any totally real algebraic field $K$. Let $\tau_{1}, \cdots, \tau_{l}$ be a set of variables in the upper half-plane. The modular group in $K$ consists of all the substitutions

$$
\tau_{k}^{\prime}=\frac{\alpha_{k} \tau_{k}+\beta_{k}}{\gamma_{k} \tau_{k}+\delta_{k}} \quad(k=1, \cdots, l),
$$

for which $\alpha, \beta, \gamma, \delta$ are integers of $K$ and $\alpha \delta-\beta \gamma$ is a totally positive unit. If $\epsilon$ 
is any unit of $K$, then $\epsilon \alpha, \epsilon \beta, \epsilon \gamma, \epsilon \delta$ define of course the same modular substitution as $\alpha, \beta, \gamma, \delta$.

Blumenthal has proved the existence of a fundamental domain $G_{0}$ of the modular group in the space of the upper half-planes of the $l$ complex variables $\tau_{k}=t_{k}+i u_{k}(k=1, \cdots, l)$. The domain $G_{0}$ is bounded by a finite number of algebraic surfaces. Since the special modular substitutions with $\alpha \delta-\beta \gamma=1$ form a subgroup of finite index $m$, they possess also a fundamental domain $G$. The non-euclidean volume

$$
V=\int \frac{d t_{1} d u_{1}}{u_{1}^{2}} \cdots \frac{d t_{l} d u_{l}}{u_{l}^{2}}
$$

of $G$, and hence also the corresponding volume $m^{-1} V$ of $G_{0}$, can be found in the following manner.

Formula (16) gives the volume

$$
v=\int_{F} d \mathfrak{X}_{1} \cdots d \mathfrak{X}_{l}
$$

for the space of the reduced systems of positive definite symmetric matrices $\mathfrak{X}_{1}, \cdots, \mathfrak{X}_{l}$ of $n$ rows with $\left|\mathfrak{X}_{1} \ldots \mathfrak{X}_{l}\right| \leqq q$. Let $n$ have the value 2 and consider instead of the group of all unimodular matrices $\left(\begin{array}{r}\alpha \delta \\ \gamma \delta\end{array}\right)$ only the subgroup for which $\alpha \delta-\beta \gamma=\epsilon^{2}$ is a square of a unit of $K$. Since the index is $2^{l}$, the corresponding volume in the space of $\mathfrak{X}_{1}, \cdots, \mathfrak{X}_{l}$ is $2^{l} v_{v}$. By the substitutions

$$
\begin{aligned}
\mathfrak{X}_{k}=\left(\begin{array}{ll}
x_{k} & y_{k} \\
y_{k} & z_{k}
\end{array}\right), \quad \tau_{k}=\frac{y_{k}+\left(y_{k}^{2}-x_{k} z_{k}\right)^{1 / 2}}{z_{k}}, \quad \xi_{k}=x_{k} z_{k}-y_{k}^{2} \\
(k=1, \cdots, l)
\end{aligned}
$$

the equation (18) is transformed into

$$
2^{l} v=\int \cdots \int\left(\xi_{1} \cdots \xi_{l}\right)^{1 / 2} d \xi_{1} \cdots d \xi_{l} \frac{d t_{1} d u_{1}}{u_{1}^{2}} \cdots \frac{d t_{l} d u_{l}}{u_{l}{ }^{2}},
$$

where the variables $t_{1}, \cdots, u_{l}$ run over $G$ and $\xi_{1}, \cdots, \xi_{l}$ over a fundamental region in $\xi_{1} \cdots \xi_{l} \leqq q$ with respect to the group $\xi^{\prime}=\epsilon^{4} \xi$ formed by the fourth powers of all units of $K$. Hence

$$
2^{l} v=\frac{1}{3} 2^{2 l-1} R q^{3 / 2} V,
$$

and by (16)

$$
V=2 \pi^{-l} D^{3 / 2} \zeta_{\star}(2) .
$$

Since it can be shown that $\pi^{-2 l} D^{1 / 2} \zeta_{k}(2)$ is rational for totally real $K$, the 
number $\pi^{-l} V$ is rational also, corresponding to a theorem of Dehn and Poincaré on the volume of a non-euclidean polyhedron in an even number of dimensions.

5. The very simple result (19) can also be proved by another method, which does not use the properties of the units and of the zeta functions of $K$. Let $\eta_{1}, \cdots, \eta_{l}$ be any positive numbers, $\mu$ and $\nu$ two numbers of $K$, not both 0 , and the ideal $(\mu, \nu)=a$. Consider the integral

$$
I(\mathfrak{a})=\sum_{(\mu, \nu)-\mathfrak{a}} \int_{G} \frac{\eta_{1} d t_{1} d u_{1}}{\left(\eta_{1}\left|\mu_{1} \tau_{1}+\nu_{1}\right|^{2}+u_{1}\right)^{2}} \cdots \frac{\eta_{l} d t_{l} d u_{l}}{\left(\eta_{l}\left|\mu_{l} \tau_{l}+\nu_{l}\right|^{2}+u_{l}\right)^{2}}
$$

where $\mu, \nu$ run over all pairs with the greatest common divisor a. If $\alpha, \beta, \gamma, \delta$ run over all integers with $\alpha \delta-\beta \gamma=1$, then $\mu \alpha+\nu \gamma, \mu \beta+\nu \delta$ run over all pairs which have the same greatest common divisor as the fixed numbers $\mu, \nu$. Moreover, if in particular $\mu \alpha+\nu \gamma=\mu, \mu \beta+\nu \delta=\nu$, then the integrand in (20) is invariant under the modular substitution $(\alpha \tau+\beta) /(\gamma \tau+\delta)$. Hence $I(\mathfrak{a})$ is exactly twice the value of the integral with the same integrand and fixed $\mu, \nu$ extended over a fundamental region for the subgroup $\Gamma$ of the modular substitutions with $\mu \alpha+\nu \gamma=\mu, \mu \beta+\nu \delta=\nu$. Choose now two numbers $\kappa, \lambda$ of the ideal $\mathfrak{a}^{-1}$; such that $\kappa \nu-\lambda \mu=1$, and make the substitution

$$
\tau^{\prime}=\frac{\kappa \tau+\lambda}{\mu \tau+\nu} .
$$

A simple calculation shows that, for all elements $\left(\begin{array}{r}\alpha \delta \\ \gamma \delta\end{array}\right)$ of $\Gamma$, the equation

$$
\left(\begin{array}{ll}
\kappa & \lambda \\
\mu & \nu
\end{array}\right)\left(\begin{array}{ll}
\alpha & \beta \\
\gamma & \delta
\end{array}\right)\left(\begin{array}{ll}
\kappa & \lambda \\
\mu & \nu
\end{array}\right)^{-1}=\left(\begin{array}{ll}
1 & \zeta \\
0 & 1
\end{array}\right)
$$

holds, where $\zeta$ belongs to $\mathfrak{a}^{-2}$; on the other hand, if $\zeta$ belongs to $\mathfrak{a}^{-2}$, then $\left(\begin{array}{c}\alpha \beta \\ \gamma \delta\end{array}\right)$ is an element of $\Gamma$. Let $\omega_{1}, \cdots, \omega_{l}$ be a basis of $a^{-2}$ and $t=\omega_{1} x_{1}+\cdots+\omega_{l} x_{l}$. Then a fundamental region of $\Gamma$ is defined by the inequalities

$$
0 \leqq x_{k}<1, u_{k}>0 \quad(k=1, \cdots, l) .
$$

Hence

$$
I(\mathfrak{a})=2 N \mathfrak{a}^{-2} D^{1 / 2} \prod_{k=1}^{l} \int_{0}^{1} \int_{0}^{\infty} \frac{\eta_{k} d x_{k} d u_{k}}{\left(\eta_{k}+u_{k}\right)^{2}}=2 N \mathfrak{a}^{-2} D^{1 / 2},
$$

and, summing (20) over all integer ideals $a$,

(21) $2 D^{1 / 2} \zeta_{k}(2)=\sum_{\mu, \nu \neq 0,0} \int_{G} \frac{\eta_{1} d t_{1} d u_{1}}{\left(\eta_{1}\left|\mu_{1} \tau_{1}+\nu_{1}\right|^{2}+u_{1}\right)^{2}} \cdots \frac{\eta_{l} d t_{l} d u_{l}}{\left(\eta_{l}\left|\mu_{l} \tau_{l}+\nu_{l}\right|^{2}+u_{l}\right)^{2}}$, 
where $\mu, \nu$ run over all pairs of integers different from 0,0 . If $\eta_{1}, \cdots, \eta_{l}$ tend to zero, the right side of (21) becomes

$$
D^{-1} \int_{G} \prod_{k=1}^{l}\left(\int_{-\infty}^{+\infty} \int_{-\infty}^{+\infty} \frac{d \mu_{k} d \nu_{k}}{\left(\left|\mu_{k} \tau_{k}+\nu_{k}\right|^{2}+u_{k}\right)^{2}} d t_{k} d u_{k}\right)
$$

where $\mu_{k}, \nu_{k}$ are variables of integration. Now, by the substitutions

$$
\begin{gathered}
\mu|\tau|+\nu \frac{t}{|\tau|}=r^{1 / 2} \cos \phi, \quad \nu \frac{u}{|\tau|}=r^{1 / 2} \sin \phi \\
\int_{-\infty}^{+\infty} \int_{-\infty}^{+\infty} \frac{d \mu d \nu}{\left(|\mu \tau+\nu|^{2}+u\right)^{2}}=\int_{0}^{\infty} \int_{0}^{2 \pi} \frac{\frac{1}{2} d r d \phi}{u(r+u)^{2}}=\pi u^{-2}
\end{gathered}
$$

and therefore the expression (22) has the value

$$
D^{-1} \pi^{l} \int_{G} \prod_{k=1}^{l} \frac{d t_{k} d u_{k}}{u_{k}^{2}}=D^{-1} \pi^{l} V
$$

Together with (21), this completes the second proof of (19).

INSTITUTE For AdVANCEd StUdy,

Princeton, N. J. 